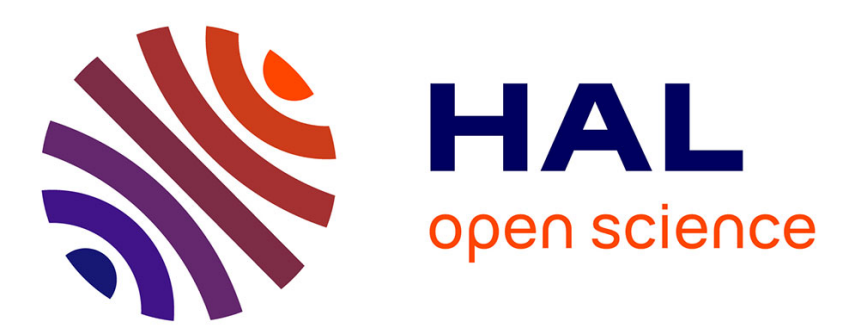

\title{
Virtual customers in sensitivity and light traffic analysis via Campbell's formula for point processes
}

François Baccelli, Pierre Bremaud

\section{To cite this version:}

François Baccelli, Pierre Bremaud. Virtual customers in sensitivity and light traffic analysis via

Campbell's formula for point processes. [Research Report] RR-1294, INRIA. 1990. inria-00075265

\section{HAL Id: inria-00075265 \\ https://hal.inria.fr/inria-00075265}

Submitted on 24 May 2006

HAL is a multi-disciplinary open access archive for the deposit and dissemination of scientific research documents, whether they are published or not. The documents may come from teaching and research institutions in France or abroad, or from public or private research centers.
L'archive ouverte pluridisciplinaire HAL, est destinée au dépôt et à la diffusion de documents scientifiques de niveau recherche, publiés ou non, émanant des établissements d'enseignement et de recherche français ou étrangers, des laboratoires publics ou privés. 


\section{Rapports de Recherche}

UNITÉ DE RECHERCHE NRIA-SOPHIA ANTIPOLIS

$$
\mathbf{N}^{\circ} 1294
$$

\section{Programme 2}

Structures Nouvelles d'Ordinateurs
Institut National de Recherche

en Informatique et en Automatique

Domaine de Voluceau

Rocquencourt

B.P.105

78153 Le Chesnay Cedex

France

Tél.:(T) 39635511

\section{VIRTUAL CUSTOMERS IN SENSITIVITY AND LIGHT TRAFFIC ANALYSIS VIA CAMPBELL'S FORMULA FOR POINT PROCESSES}

François BACCELLI

Pierre BRÉMAUD

Octobre 1990 


\title{
Virtual Customers in Sensitivity and Light Traffic Analysis via Campbell's Formula for Point Processes.
}

\author{
by \\ F. Baccelli ${ }^{1}$ and P. Brémaud ${ }^{2}$ \\ 1: INRIA Sophia-Antipolis France \\ 2: Laboratoire des Signaux et Systèmes, Gif sur Yvette France
}

July 1990

Abstract: This article provides the theoretical basis of the virtual customer method or positive rare perturbation (RPA) method of sensitivity analysis and in particular gives a short proof of the light traffic derivative result of Reiman and Simon [4].

Kieywords: Sensitivity analysis, Light traffic, Queues, Point processes. 


\section{Introduction}

Sensitivity analysis and light traffic analysis are concerned with the computation of derivatives with respect to a parameter of performance measures of discrete event systems occurring for instance in flexible manufacturing or in queueing networks.

In the case of light traffic theory, the derivative is computed at a value of the parameter for which the average intensity of a given flow of events is null. A typical example is the computation of the average workload derivative of a GI/GI/1/ $\infty / F C F S$ quene with respect to $\theta$ for $\theta=\theta_{0}$ in the case where the interarrival time goes to $\infty$ in distribution as $\theta$ tends to $\theta_{0}$. In light traffic theory the computation of derivatives of first and higher orders leads to explicit results in closed form, whereas in sensitivity analysis. the derivatives are given under the form of an expectation which is then evaluated by the standard methods of simulation. In sensitivity analysis the derivatives are computed at a value of the parameter $\theta$ for which, in general, flows of events have positive average intensity.

In the landmark article of Reiman and Simon [4], a direct analytic method leads to formulas that can be interpreted in terms of virtual customers. In sensitivity analysis, the method which has received most of the attention is the perturbation method of Ho et alii ([3], [5] for instance). It is called the IPA method (infinitesimal perturbation analysis) and is based on the infinitesimal perturbation of a single trajectory. There is an altemative method called the RPA method (rare perturbation analysis) in Brémaud [2]. The RPA method of [2] is of the negative type: it consists of "phantomizing" one customer per cycle, by comparing the nominal system with the sane systen with the phantom custoner removed. As mentioned in [2], there is a clual RPA method, where the nominal system is augmented by one virtual customer per cycle. The phantom RPA method was called the negative RPA method in [2], whereas the virtual RPA method was named the positive RPA method.

The light traffic analysis of Reiman an Simon [4] will be revisited in the present inticle as a special case of the virtual custoner RPA method. However our analysis will be different from that of [2]. It will be shown in particular that the negative 
RPA formula and the light traffic derivative formula can be obtained in a very simple manner by using Campbell's formula for point processes (see [1] for instance).

This new approach has to be compared in the light traffic theory to the analytic approach of Reiman and Simon [4]. First of all, we do not require a priori a condition on the basic functional because there are several inconveniences to this approach: first of all it seems to require a complicated proof, and secondly it does not stay close enough to generality in the sense that one might very well loose systems just because they do not verify the assumption when these systems could be otherwise analyzed. In our approach, we make a simple use of Campbell's theorem for point processes and impose an "ad hoc" condition just to make things work. The "ad hoc" condition is always readily checked at least for all cases that have been considered in [4] for instance. One should notice that in the approach of Reiman and Simon, the fundamental assumption must be checked for every system, therefore showing no advantage on our "ad hoc" condition. Also, our argument is purely probabilistic and involves only one limiting procedure, the one essential to the problem, that is: the intensity vanishes.

Our method of proof easily extends to the "computation" of derivatives at non null intensities, and provides theoretical ground for the positive RPA method, or the virtual customer method of sensitivity analysis. In addition it easily extends, without further work, to the computation of derivatives of the first order when the flow of events is not Poisson.

\section{Derivatives at Null Intensity: the Poisson Case}

Let $V$ be a Poisson process of intensity $\triangle \lambda$ defined on $\Re$, and let $\left\{T_{n}\right\}, n \in Z$, be its sequence of event times with the convention $\quad \cdots<T_{-1}<T_{0} \leq 0<T_{1}<T_{2} \cdots$. Let $\left\{Z_{n}\right\}, n \in Z$, be an iid sequence of random marks taking their values in some measurable space $(E, \Xi)$. The processes $N$ and $\left\{Z_{n}\right\}$ are assumed independent.

Call $P_{\triangle \lambda}$ the underlying probability measure. 
Let $\psi$ be a functional of $\left(N,\left\{Z_{n}\right\}\right)$ of the form

$$
\dot{\psi}=h\left(T_{0}, Z_{0}, \tau_{-1}, Z_{-1}, \tau_{-2}, Z_{-2}, \cdots\right)
$$

where $\tau_{n}=T_{n+1}-T_{n}$ is the $n$th time interval of $N$. The objective of this paper is to compute

$$
\lim _{\triangle \lambda \downarrow 0} \frac{1}{\Delta \lambda} \quad E_{\Delta \lambda}[\psi]
$$

Assuming that the functional $\psi\left(N,\left\{Z_{n}\right\}\right)$ is null if the trajectory $N$ has no points, the quantity (2) is the right-hand derivative at time $\lambda=0$ of $E_{\lambda}[\psi]$.

Before stating the conditions under which the derivative (2) will be computed a few notations have to be introduced. Define the functional $g$ by

$$
\begin{aligned}
& g\left(T_{n}, Z_{n}, \tau_{n-1}, Z_{n-1}, \tau_{n-2}, Z_{n-2}, \cdots\right) \\
& =h\left(T_{n}, Z_{n}, \tau_{n-1}, Z_{n-1}, \cdots\right)-h\left(T_{n}-\tau_{n-1}, Z_{n-1}, \tau_{n-2}, Z_{n-2}, \cdots\right) .
\end{aligned}
$$

In order to shorten the notations, write the left-hand side of the above identity $g\left(T_{n}, Z_{n}, Y_{n-1}\right)$ where $Y_{n-1}=\left(\tau_{n-1}, Z_{n-1}, \tau_{n-2}, Z_{n-2}, \cdots\right)$. Also for $\alpha>0$, define $Y_{n-1}^{a}=\left(\alpha \tau_{n-1}, Z_{n-1} ; \alpha \tau_{n-2}, Z_{n-2}, \cdots\right) ; Y_{n-1}^{a}$ is therefore obtained from $Y_{n-1}$ by dilation of the sequence $\left(\tau_{n-1}, \tau_{n-2}, \cdots\right)$ by $\alpha$.

The assumptions on $\psi$ are the following.

(A1) For some $\alpha_{0}>0$

$$
\int_{-\infty}^{0} E_{1}\left[\left|g\left(t, Z_{0}, Y_{-1}^{\alpha}\right)\right|\right] d t<\quad \infty \text { for } \quad \alpha \geq \alpha_{0}
$$

(42) There exists a functional $g_{\infty}\left(t, Z_{0}\right)$, not depending on $Y_{-1}$, such that

$$
\lim _{\substack{\alpha \uparrow \infty \\ \alpha \geq \alpha_{0}}} \int_{-\infty}^{0} E_{1}\left[\left|g\left(t, Z_{0}, Y_{-1}^{-a}\right)-g \infty\left(t, Z_{0}\right)\right|\right] d t=0
$$

Theorem 1. Under conditions (A1) and (A2),

$$
\lim _{\triangle \lambda \mid U} \frac{1}{\Delta \lambda} E_{\triangle \lambda}[\psi]=\int_{-\infty}^{0} E_{1}\left[g_{\infty}\left(t, Z_{0}\right)\right] d t
$$


Remark 1. Assumption A1 is equivalent to

$$
\int_{-\infty}^{0} E_{\lambda}\left[\left|g\left(t, Z_{0}, Y_{-1}\right)\right|\right] d t \quad<\infty \quad \text { for } \quad \lambda \leq \frac{1}{\alpha_{0}}
$$

By Campbell's formula

$$
\lambda \int_{-\infty}^{0} E_{\lambda}\left[\left|g\left(t, Z_{0}, Y_{-1}\right)\right|\right] d t=E_{\lambda}\left[\sum_{n \leq 0}\left|g\left(T_{n}, Z_{n}, Y_{n-1}\right)\right|\right]
$$

and therefore, for $\lambda \leq \frac{1}{\alpha_{0}}, \sum_{n \leq 0} g\left(T_{n}, Z_{n}, Y_{n-1}\right)$ converges $P_{\lambda} \cdot$ a.s. and is equal to 4. Thus (A1) implies that, $E_{\lambda}[|\psi|]<\infty$ for $\lambda \leq \frac{1}{\alpha_{0}}$. $\diamond$

Remark 2. Since $(t, \omega) \rightarrow g_{\infty}\left(t . Z_{o}(\omega)\right)$ is a $L_{1}$ limit (for the measure $d P_{1} \times$ d.t $), E_{1}\left[\int_{-\infty}^{u}\left|g\left(t, Z_{0}\right)\right|\right] d t<\infty$ and therefore the quantity $\int_{-\infty}^{0} E_{1}\left[g\left(t, Z_{0}\right)\right] d t$ is well defined. $\diamond$

Renark 3. Going back to the expression (3), one sees that the obvious choice of $y_{x}$ is

$$
g_{\infty}\left(t, Z_{0}\right)=\lim _{\alpha \mid x}\left\{h\left(t . Z_{0}, \alpha \tau_{-1} . Z_{-1}, \cdots\right)-h\left(t-\alpha \tau_{-1}, Z_{-1}, \cdots\right)\right\}
$$

The physical interpretation of $g_{\infty}\left(t, Z_{0}\right)$ is that of the value of the functional $\psi$ when there arrived only one customer before time 0 (this customer arriving at time $T_{0}=t$, and being marked by $\left.Z_{0}\right)$. $\diamond$

Proof of tla theorem. From Campleclls formula and Remark 1

$$
\begin{aligned}
\frac{1}{\lambda} E_{\lambda}[\psi] & =\frac{1}{\lambda} E_{\lambda}\left[\sum_{n \leq 0} g\left(T_{n}, Z_{n}, Y_{n-1}\right)\right] \\
& =E_{\lambda}\left[\int_{-\infty}^{0} g\left(t, Z_{0}, Y_{-1}\right) d t\right]=\int_{-\infty}^{0} E_{1}\left[g\left(t, Z_{0}, Y_{-1}^{-1}\right)\right] d t
\end{aligned}
$$

for $a=\frac{1}{\lambda}$. The theorem inmediately follows from this observation and Assumption $42 . \diamond$ 
Example 1: The workload of $\mathrm{M} / \mathrm{GI} / 1 / \infty / \mathrm{FCFS}$. Interpret $T_{n}$ as the arrival of a customer with required service $Z_{n}=\sigma_{n}$ into a $/ / 1 / \infty /$ FCFS queueing system. Take $\psi$ to be the workload at time $t=0$ in the stationary regime $\left(\lambda<E\left[\sigma_{1}\right]^{-1}\right)$. Observe that

$$
\psi=\sum_{n \leq 0} f\left(T_{n}, \sigma_{n}, W_{n}\right)
$$

where $W_{n}$ is the waiting time of customer $n$ and

$$
f\left(T_{n}, \sigma_{n}, W_{n}\right)= \begin{cases}\sigma_{n} & \text { if } T_{n}+W_{n} \geq 0 \\ 0 & \text { if } T_{n}+W_{n}<-\sigma_{n} \\ \sigma_{n}+T_{n}+W_{n} & \text { if }-\sigma_{n} \leq T_{n}+W_{n}<0\end{cases}
$$

Therefore since $W_{n}=v\left(\tau_{n-1}, \sigma_{n-1}, \cdots\right)=v\left(Y_{n-1}\right)$ for some functional $v$,

$$
g\left(T_{n}, \sigma_{n}, Y_{n-1}\right)=f\left(T_{n}, \sigma_{n}, v\left(Y_{n-1}\right)\right)
$$

Also

$$
g\left(t, \sigma_{0}, Y_{1}^{-\alpha}\right)=f\left(t, \sigma_{0}, W_{0}^{\alpha}\right)
$$

where $W_{0}^{\alpha}$ is the waiting time of customer 0 corresponding to the data $\alpha \tau_{-1}, \sigma_{-1}$, $\alpha \tau_{-2}, \sigma_{-2}, \cdots$. Since at equilibrium the workload is null on a set of times of infinite Lebesgue measure, it is clear that $W_{0}^{\alpha}$ decreases and eventually becomes null for a finite a (depending on the trajectory). Therefore

$$
g_{\infty}\left(t, \sigma_{0}\right)=f\left(t, \sigma_{0}, 0\right)=\left(\sigma_{0}+t\right)^{+}
$$

Condition $A 1$ is guaranteed by $E\left[\sigma_{0}^{2}\right]<\infty$. As for assumption A2, it follows by Lebesgue's theorem. Finally, since

$$
\int_{-\infty}^{0} E\left[\left(\sigma_{0}+t\right)^{\dagger}\right] d t=\frac{1}{2} E\left[\sigma_{0}^{2}\right]
$$

the result is

$$
\frac{d}{d \lambda} E_{\lambda}[\psi]=\frac{1}{2} E\left[\sigma_{0}^{2}\right]
$$


as car be directly checked on Pollaczek-Khintchine's formula. $\diamond$

\section{Derivative at Positive Intensity and Higher Order Derivatives at 0: the Poisson Case}

Let $N$ and $\left\{Z_{n}\right\}$ be as before. Let $\tilde{N}$ and $\left\{\tilde{Z}_{n}\right\}$ have the same statistics as $N,\left\{Z_{n}\right\}$ except that the intensity of the Poisson process $\tilde{N}$ is $\lambda>0,\left(N,\left\{Z_{n}\right\}\right)$ and $\left(\tilde{N},\left\{\tilde{Z}_{n}\right\}\right)$ are assumed to be independent. If one merges the input flows $\left(N,\left\{Z_{n}\right\}\right)$ and $\left(\tilde{N},\left\{\tilde{Z}_{n}\right\}\right)$ one obtains an input flow $\left(N^{\prime},\left\{Z_{n}^{\prime}\right\}\right)$ of the same type, only with $N^{\prime}$ being of intensity $\lambda+\Delta \lambda$.

Call $P_{\lambda, \Delta \lambda}$ the underlying probability measure.

The functional $\psi$ is now of the form

$$
\psi=h\left(T_{0}, Z_{0}, \tau_{-1}, Z_{-1}, \cdots ;\left\{\tilde{T}_{n}\right\},\left\{\tilde{Z}_{n}\right\}\right)
$$

where for $V=\emptyset$, we denote $\psi=h\left(\emptyset ;\left\{\tilde{T}_{n}\right\},\left\{\tilde{Z}_{n}\right\}\right)$. The objective is now to find an expression for

$$
\lim _{\Delta \lambda j 0} \frac{1}{\Delta \lambda}\left[E_{\lambda, \Delta \lambda}[\dot{\psi}]-E_{\lambda, 0}[\psi]\right]
$$

provided this limit exists.

The same notations as before are used, except that there is now an extra dependency on $\tilde{N},\left\{\tilde{Z}_{n}\right\}$, and that the following assumptions are made:

(B1) Fir sonic $a_{0}>0$

$$
\int_{-\infty}^{0} E_{\lambda, 1}\left[\mid g\left(t, Z_{0}, Y_{-1}^{-\alpha}, \tilde{N}_{,},\left\{\tilde{Z}_{n}\right\} \mid\right]<\infty \text { for all } \alpha \geq \alpha_{0}\right.
$$

(B2) There exists a functional $g_{\infty}\left(t, Z_{0}, \hat{N},\left\{\tilde{Z}_{n}\right\}\right)$ not depending on $Y_{-1}^{-}$, such that

$$
\lim _{\substack{a t \infty \\ a \geq u_{0}}} \int_{-\infty}^{u} E_{\lambda, 1}\left[\left|g\left(t, Z_{0}, Y_{-1}^{-c r} ; \tilde{N},\left\{\tilde{Z}_{n}\right\}\right)-g_{\infty}\left(t, Z_{0} ; \tilde{N},\left\{\tilde{Z}_{n}\right\}\right)\right|\right] d t=0 .
$$

Theorem 2. Linder conditions (B1) and (B2)

$$
\lim _{\triangle \lambda \mid . \cup} \frac{1}{\Delta \lambda}\left\{E_{\lambda, \Delta \lambda}\left[\psi^{\prime}\right]-E_{\lambda, u}\left[\psi_{i}\right]\right\}=\int_{-\infty}^{0} E_{\lambda, 1}\left[g_{\infty}\left(t, Z_{0} ; \tilde{N},\left\{\tilde{Z}_{n}\right\}\right)\right] d t
$$


Proof: same as Theorem $1 . \diamond$

Remark 4. The interpretation of $g_{\infty}\left(t, Z_{0} ; \tilde{N},\left\{\tilde{Z}_{n}\right\}\right)$ is that it is the value of $\psi$ computed with the input flow $\tilde{N},\left\{\tilde{Z}_{n}\right\}$ plus a customer at $t$ with an attribute mark $Z_{0} . \diamond$

Higher order derivatives: From Theorems 1 and 2

$$
\begin{array}{r}
\frac{d}{d \lambda} E_{\lambda}[\psi]-\left(\left.\frac{d}{d \lambda} E_{\lambda}[\psi]\right|_{\lambda=0}\right)= \\
E_{\lambda}\left[\int_{-\infty}^{0} E\left[g_{\infty}\left(t, Z_{0} ; \tilde{N},\left\{\tilde{Z_{u}}\right\}\right)-g_{\infty}\left(t, Z_{0}\right) \mid \tilde{N},\left\{\tilde{Z_{n}}\right\}\right] d t\right]=E_{\lambda}[\Phi]
\end{array}
$$

where

$$
\Phi=\int_{-\infty}^{\prime \prime} E\left[g_{\infty}\left(t, Z_{0} ; \tilde{N},\left\{\tilde{Z}_{n}\right\}\right)-g_{\infty}\left(t, Z_{0}\right) \mid \tilde{N},\left\{\tilde{Z}_{n}\right\}\right] d t
$$

In order to compute, if it exists, the second derivative at $\lambda=0$ of $E_{\lambda}[\psi]$, i.e.

$$
\lim _{\lambda] 0} \frac{1}{\lambda} E_{\lambda}[\Phi]=\left.\frac{d^{2}}{d \lambda^{2}} E_{\lambda}[\psi]\right|_{\lambda=0}
$$

one can use again Theorem 1.

\section{First Order Derivative: the non-Poisson Case}

Consider the situation described at the beginning, except that now $\left\{T_{n}\right\}$ is not required to be Poisson. It is required to be a stationary renewal point process with intensity $\lambda(\theta)$ where $\theta \in \Re_{+}$is a parameter governing the underlying probability $P_{\theta}$. It is still assumed that $N$ and $\left\{Z_{n}\right\}$ are $P_{\theta}$-independent.

We are interested in computing

$$
\lim _{\theta \mid 0} \frac{1}{\theta} E_{\theta}[\psi]
$$

Assuming that $E_{0}[\psi]=0$, the cuantity $(11)$ is the derivative of $E_{\theta}[\psi]$ with respect to $\theta$, at $0+$. 
The aswm ntions on \& are:

(C1) For some $\theta_{0}>0$,

$$
\int_{-\infty}^{0} E_{\theta}\left[\left|g\left(t, Z_{0}, Y_{-1}\right)\right|\right] d t<\infty \text { for all } \theta \leq \theta_{0} \text {. }
$$

(C'2) There exists a functional $g_{0}\left(t, Z_{0}\right)$, not depending on $Y_{-1}$, such that

$$
\lim _{\substack{j, 0 \\ 0 \leq \rho_{0}}} \int_{-\infty}^{0} E_{\theta}\left[\left|g\left(t, Z_{0}, Y_{-1}\right)-g_{0}\left(t, Z_{0}\right)\right|\right] d t=0
$$

Assinthe moreover

$$
\lim _{0 \leqslant 0} \frac{\lambda(\theta)}{\theta}=\lambda^{\prime}(0)
$$

Theorem 3. Uneler conditions (C1), (C2) and (C3)

$$
\lim _{\theta ! 0} \frac{1}{\theta} E_{\theta}[\psi]=\lambda^{\prime}(0) \int_{-\infty}^{0} E\left[g_{0}\left(t, Z_{0}\right)\right] d t .
$$

Prouf: smine as Theorem 1. $\diamond$

Eximple 2. Worlilond of a GI/GI/1/00/FCFS queue. The situation is as in Exanuple 1 except then now $\left\{T_{11}\right\}$ is a stationary renewal process with interarrival distribution $G_{\theta}\left(x^{\prime}\right)$ such that

$$
\lambda(\theta)=\frac{1}{\int_{0}^{\infty} x d G_{\theta}(x)}
$$

verifies $(C 3)$ and such that $\lim _{\theta \downarrow 0} P_{\theta}\left(\tau_{1} \geq a\right)=1$ for all $a>0\left(\tau_{1} \stackrel{\mathcal{D}}{\rightarrow} \infty\right.$ as $\left.\theta \downarrow 0\right)$.

Theorem T3 applies with $g_{0}\left(t, \sigma_{0}\right)=\left(\sigma_{0}+t\right)^{+}$and $g\left(t, \sigma_{0}, Y_{-1}\right)$ as in Example 1. Assumption (C'2) reacls

$$
\lim _{\theta \mid 0} \int_{-\infty}^{U} E_{\theta}\left[f\left(t, \sigma_{0}, W_{0}\right)-\left(\sigma_{0}+t\right)^{+}\right] d t=0
$$




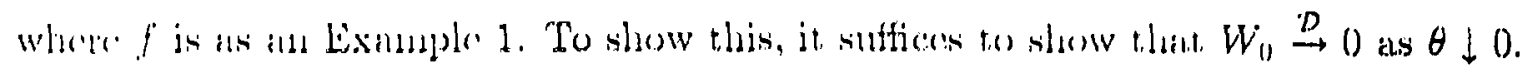
'This and be done by observing that. $W_{1} \sim W_{0}$ and that.

$$
E_{\theta}\left[e^{i n W_{1}}\right]=E_{\theta}\left[c^{i n\left(W_{0}+\sigma_{0}-\tau_{0}\right)+}\right]
$$

ancl by using tho liypothesio $\tau_{0} \stackrel{P}{\rightarrow}+\infty$ us $\theta \downarrow 0 . \diamond$

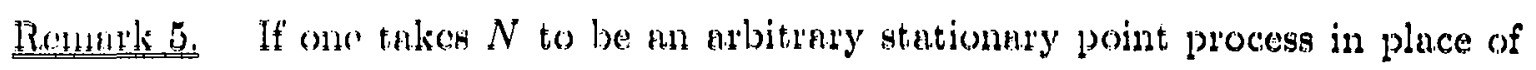
11 fouwn process, the result of Thooren 3 ulso holds provided the conditions (C1'), (C2') und (C3) hold, where (C2') and (C3') are similar to (C2) and (C3), but with En leplated by the: Palm probubility of $N, E_{\theta}^{\prime \prime} . \diamond$

\section{References}

11) Buccelli, F, and Brénaud, P. (1987) Pnlm Probability and Stationary Queueing Symteruts, Lact. Notes in Stutistics 41, Springer Vorlag, New-York.

[2] Bremancl, P. (1000) On computing clerivatives with respect to the rate of a Poisson

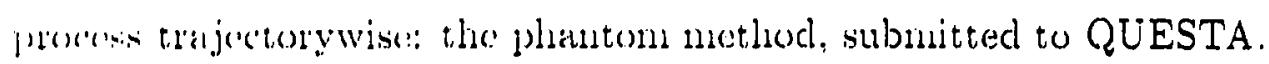

[3] Ho, Y.C. and C'ao, X.R. (10S3) Porturbation Analysis and Optimization of Queue-

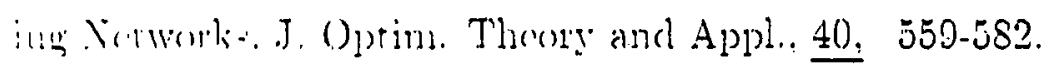

[4] Rinunu. M.I. and Simon, B. (1989) Open Queueing Systems in Light Traffic, Mirtl. of () pers. IRes.. 1t, 1. 26-59.

(-) Smi. Ri. (1080) Pratmbation Analysis: The State of the Art and Research Issues Explainad Via the CiI/GI/1 Quene. Proceedings of the IEEE, 77, 1, 114-137. 
ISSN 0249-6399 